\title{
BENFORD'S LAW AND PSYCHOLOGICAL BARRIERS IN CERTAIN eBay AUCTIONS
}

\author{
Ocean Fan Lu \& David E. Giles* \\ Department of Economics, University of Victoria \\ Victoria, B.C., Canada V8W $2 Y 2$
}

September, 2006

\begin{abstract}
Using generalizations of Benford's Law we test for the absence of psychological barriers at various price levels in eBay auctions for professional football tickets. Our empirical results indicate that this hypothesis cannot be rejected.
\end{abstract}

Keywords: $\quad$ Psychological barriers, auction prices, Benford’s law

JEL Classifications: C12, C16, D44

* We are grateful to Sophie Hesling for her assistance with data collection, and to Don Ferguson for his helpful comments.

\section{Author Contact:}

David Giles, Dept. of Economics, University of Victoria, P.O. Box 1700, STN CSC, Victoria, B.C., Canada V8W 2Y2; e-mail: dgiles@uvic.ca; FAX: (250) 721-6214 


\section{Introduction}

Several studies have investigated the possibility of psychological barriers or "resistance levels" in financial markets. Most of these studies (e.g., Donaldson and Kim, 1993; Ley and Varian, 1994; Koedijk and Stork, 1994; and Cyree et al., 1999) assume that the digits of the associated prices are uniformly distributed. However, De Ceuster et al. (1997) use Benford's Law to show that this assumption is inappropriate, and their analysis questions previous findings of such barriers.

It is natural to ask whether there are similar resistance levels in other markets, and auction price data provides one source of information to address this question. Why won't certain bidders go higher than some price level? What are the thresholds for the bids? We analyze eBay auction data for professional football tickets and find no statistical evidence of psychological barriers at a variety of levels.

The mechanism behind this research is to extract the significant digits of the auction prices to examine whether their distributions follow generalized versions of Benford's Law, which are introduced in section 2. Section 3 describes our data and the use of ' $M$-values' defined by De Ceuster et al. (1997) to capture information about the relative proximity to a psychological barrier. Our empirical test results appear in section 4, and some concluding remarks are given in section 5.

\section{Benford's Law}

Benford (1938) re-discovered Newcomb’s (1881) finding that many naturally occurring numerical data exhibit special features with regard to the first significant digit. He showed that the distribution of first significant digits is non-uniform. Benford's Law indicates that in numbers from many real-life data sources, the first significant digit " 1 ” occurs with a probability of almost $31 \%$, not $11.1 \%$ (1/9). The bigger the digit, the less likely it is to occur as the first significant digit.

Specifically, let $D_{1}$ denote the first significant digit in a number $N$. If $N=95,579.43$ then $D_{1}=9$; if $N=0.0498$, then $D_{1}=4$. Benford's Law states that

$$
\operatorname{Pr} .\left[D_{1}=k\right]=\log _{10}[1+(1 / k)] \quad ; \quad k=1,2, \ldots, 9 .
$$

What is less well known is that the joint distribution for second and higher significant digits is

$$
\operatorname{Pr} .\left[D_{1}=d_{1}, \ldots, D_{k}=d_{k}\right]=\log _{10}\left[1+\left(\sum_{i=1}^{k} d_{i} \times 10^{k-i}\right)^{-1}\right] \text {, }
$$


for $d_{i} \in\{1,2, \ldots, 9\}$ and $d_{j} \in\{0,1,2, \ldots, 9\}, j>1$. So, the probability of the first three significant digits being 5, 1 and 8 is $\log _{10}\left[1+\frac{1}{518}\right]=0.00084$. This distribution is invariant to scale (Pinkham, 1961).

Stock prices and tax data (Geyer and Williamson, 2004; Nigrini, 1992; Nigrini and Mittermaier 1997) obey Benford's Law, as do winning bids for certain eBay auctions (Giles, 2006). Benford’s Law provides a foundation for testing for "resistance levels" in markets. De Ceuster et al. (1997) proposed a test for psychological barriers using cyclical permutations of the observed daily stock returns and concluded that there was no evidence of psychological barriers in various U.S., U.K. and Japanese stock market indices. Aggarwal and Lucey (2006) analysed gold prices in a similar way and found evidence that psychological barriers at the 100's digits (price levels such as $\$ 200$, $\$ 300$, etc.).

\section{Data and M-statistics}

Giles (2006) has shown that the closing prices of successful eBay auctions for pro-football game tickets satisfy Benford's Law, which suggests the absence of market collusion among bidders or intervention by sellers. Using his data, our sample comprises all 1,159 successful auctions for tickets for professional U.S. football games in the eBay "event tickets" category between 25 November and 2 December 2004. As Benford's Law is satisfied, we can use these data to construct statistics for testing for the existence of various psychological barriers.

We denote the successful auction prices as $P_{t}, t=1, \ldots, n$, with $n=1,159$. We consider potential barriers at the levels ..., 300, 400, ..., etc. (Donaldson, 1990; Donaldson and Kim, 1993; Ley and Varian, 1994; De Ceuster et al., 1997), or:

$$
k \times 100, \quad k=1,2, \ldots ., \text { etc. }
$$

In order to represent psychological barriers at all levels, we should consider barriers at the levels ..., 10, 20,.., 100, 200,..,1000, 20000,..., etc., for

$$
k \times 10^{a}, \quad k=1,2, \ldots, 9 ; a=\ldots,-1,0,1, \ldots ;
$$

or at the more comprehensive set of levels $\ldots, 10,11, \ldots, 100,110, \ldots, 1000,1100, \ldots$, etc., for

$$
k \times 10^{a}, \quad k=10,11, \ldots, 99 ; a=\ldots,-1,0,1, \ldots .
$$

We then need to define $M$-values, which are meant to carry the information on the relative closeness to a barrier. Corresponding to the above levels defined in equation (1), 


$$
M_{t}^{a}=\left[P_{t}\right] \bmod 100,
$$

where $\left[P_{t}\right]$ is the integer part of the prices, 'mod100' stands for modulo 100 , and clearly, the $M^{a}$ values are made up of the pair of trailing digits preceding the decimal point. For barriers at the levels defined by equation (2) and (3), the $M$-values are

$$
\begin{aligned}
& M_{t}^{b}=\left[100 \times 10^{\left(\log p_{t}\right) \bmod 1}\right] \bmod 100 \\
& M_{t}^{c}=\left[1000 \times 10^{\left(\log P_{t}\right) \bmod 1}\right] \bmod 100
\end{aligned}
$$

$M^{a}$ selects the pair of digits in $P_{t}$ preceding the decimal point; $M^{b}$ selects the second and third significant digits; and $M^{c}$ picks the third and fourth significant digits. Many researchers wrongly assume that the $M$-values are uniformly distributed in the absence of psychological barrier, at least in large samples. De Ceuster et al. (1997) derived of the limit distributions of the $M$-values, which we have applied in part of the following analysis:

$$
\begin{aligned}
& \lim _{t \rightarrow \infty} \operatorname{Pr} .\left(M_{t}^{a}=k\right)=\lim _{n \rightarrow \infty} \sum_{i_{1}=1}^{9} \sum_{i_{2}=0}^{9} \cdots \sum_{i_{n-1}=0}^{9} \log \left(\frac{\sum_{r=1}^{n-1} i_{r} \times 10^{n-r+1}+k+1}{\sum_{r=1}^{n-1} i_{r} \times 10^{n-r+1}+k}\right)=\frac{1}{100} \\
& \lim _{t \rightarrow \infty} \operatorname{Pr} .\left(M_{t}^{b}=k\right)=\sum_{i=1}^{9} \log \left(\frac{i \times 10^{2}+k+1}{i \times 10^{2}+k}\right) \\
& \lim _{t \rightarrow \infty} \operatorname{Pr} .\left(M_{t}^{c}=k\right)=\sum_{i=1}^{9} \sum_{j=0}^{9} \log \left(\frac{i \times 10^{3}+j \times 10^{2}+k+1}{i \times 10^{3}+j \times 10^{2}+k}\right)
\end{aligned}
$$

The limit probabilities in equations (4), (5) and (6) give us the relative frequencies over the sample $t=1, \ldots, n$, of the $M$-values when there are no psychological barriers in the market, and the sample size $n$ tends to infinity. The frequencies for the $M$-values in our data are expected to be non-uniform for $M_{t}^{b}$ and $M_{t}^{c}$, and uniform for $M_{t}^{a}$. Although the uniformity of $M_{t}^{a}$ seems to rationalize the standard assumption of uniformity testing for the presence of psychological barriers, $M_{t}^{a}$ can only capture psychological barriers at the levels $\ldots, 200,300, \ldots, 3400$, $3500, \ldots .$. etc., and the series at those levels is not multiplicatively regenerative (De Ceuster et al., 1997). Therefore, results that are obtained from investigating only the $M_{t}^{a}$ values could lead to the wrong conclusion, and it is crucial that the $M_{t}^{b}$ and $M_{t}^{c}$ values are also considered. 


\section{Test results}

First, we test whether our data exhibit properties consistent with the limit distributions in equations (4) - (6). The null hypothesis is that there are no psychological barriers in prices for pro-football tickets in the eBay auction market. The Kolmogorov-Smirnov (K-S) test and other non-parametric tests such as "integrated deviations" tests of the Cramér-von Mises type are not appropriate here because of the "circular” nature of our data (Giles, 2006). Kuiper's test is similar to the familiar K-S test, which uses the difference statistics $D_{n}^{+}$and $D_{n}^{-}$. Kuiper's test combines $D_{n}^{+}$and $D_{n}^{-}$into one statistic, making the test as sensitive in the tails as at the median, and making it invariant under cyclical data transformations. If the empirical distribution function for the sample is $F_{n}(x)$, and the null population distribution function is $F_{0}(x)$, Kuiper's test statistic is:

$$
V_{n}=D_{n}^{+}+D_{n}^{-},
$$

where

$$
\begin{aligned}
& D_{n}^{+}=\sup _{-\infty<x<\infty}\left(F_{n}(x)-F_{0}(x)\right) \\
& D_{n}^{-}=\sup _{-\infty<x<\infty}\left(F_{0}(x)-F_{n}(x)\right) .
\end{aligned}
$$

Another important feature of Kuiper's test is that the null distribution of the test statistic is invariant to the hypothesized distribution, for all $n$. Stephens (1970) tabulates critical values for the null distribution of the transformed statistic

$$
V_{n}^{*}=V_{n}\left(n^{1 / 2}+0.155+0.24 n^{-1 / 2}\right)
$$

The $10 \%, 5 \%$ and $1 \%$ critical values are $1.620,1.747$ and 2.001 respectively, for all $n$. Our $V_{n}^{*}$ values for $M^{a}, M^{b}$ and $M^{c}$ are $0.947,1.663$ and 4.022 respectively. So, our values for $M^{a}$ and $M^{b}$ are consistent with the hypothesis of no psychological barriers, at the $5 \%$ level, but our value for $M^{c}$ implies a rejection of this hypothesis against the most general of the alternative hypotheses considered.

Our goodness-of-fit tests are designed to examine whether the empirical data have characteristics consistent with the limit distributions for the $M$-values discussed above. De Ceuster et al. (1997) 
show that extremely large sample sizes are needed for these limit distributions to be applicable, and the finite-sample distributions are data-dependent.

We follow De Ceuster et al. (1998) and also test for psychological barriers using cyclical permutations of the data. Psychological barriers generate an abnormally low number of $M$-values in the " 00 " region. Let $\Psi$ be a set of $M$-values in the neighbourhood of a psychological barrier, such as $\Psi=\{00\}, \Psi=\{99,00,01\}$, etc. For some $\Psi$, the auction price $P_{t}$ is in a neighbourhood of a psychological barrier if $M_{t} \in \Psi$. Let $I_{\Psi}\left(M_{t}\right)=1$ in this case, zero otherwise, and define $\psi=\sum_{t=1}^{n} I_{\Psi}\left(M_{t}\right)$. For any $M$ and $\Psi$, a sufficiently small $\psi$ signals a psychological barrier. The distribution of $\psi$ is data-specific, but p-values for the hypothesis of no psychological barrier can be bootstrapped as the proportion of bootstrapped $\psi$ values less than the empirical $\psi$. Our experimental results, for 10,000 repetitions, appear in Table 1 . The null hypothesis of no psychological barriers in the auction prices for football tickets cannot be rejected.

\section{Conclusions}

Using some generalizations of Benford's Law we find that psychological barriers are absent in eBay auctions for pro-football tickets. This is consistent with De Ceuster et al.'s (1997) finding various stock indices, but contrasts with Aggarwal and Lucey 's (2006) results for gold prices. Our results have implications for users of eBay’s “proxy bidding” service. For example, offering a maximum bid of $\$ 100.01$ in anticipation that opponents have a psychological barrier at or just under $\$ 100$ may not be a viable strategy. It would be interesting to extend this analysis to other eBay categories. 
Table 1: Bootstrap Results

\begin{tabular}{|c|c|c|c|c|}
\hline & $\Psi$ & $\psi$-Statistic & $\begin{array}{l}\text { Bootstrap } \\
\text { Mean }\end{array}$ & $\begin{array}{l}\text { p-Value } \\
(\%)\end{array}$ \\
\hline \multirow[t]{6}{*}{$\boldsymbol{M}^{a}$} & $\{00\}$ & 51 & 51.01 & 48.07 \\
\hline & $\{99, \ldots ., 01\}$ & 71 & 71.03 & 48.20 \\
\hline & $\{98, \ldots, 02\}$ & 124 & 124.12 & 47.94 \\
\hline & $\{97, \ldots, 03\}$ & 133 & 133.12 & 48.11 \\
\hline & $\{95, \ldots, 05\}$ & 190 & 190.18 & 48.42 \\
\hline & $\{90, \ldots, 10\}$ & 301 & 301.16 & 48.02 \\
\hline \multirow[t]{6}{*}{$M^{b}$} & $\{00\}$ & 96 & 95.97 & 48.81 \\
\hline & $\{99, \ldots ., 01\}$ & 121 & 121.03 & 49.13 \\
\hline & $\{98, \ldots, 02\}$ & 174 & 174.12 & 48.53 \\
\hline & $\{97, \ldots, 03\}$ & 183 & 183.14 & 48.76 \\
\hline & $\{95, \ldots \ldots 05\}$ & 247 & 247.25 & 47.86 \\
\hline & $\{90, \ldots ., 10\}$ & 409 & 409.09 & 48.96 \\
\hline \multirow[t]{6}{*}{$\boldsymbol{M}^{c}$} & $\{00\}$ & 445 & 444.84 & 49.04 \\
\hline & $\{99, \ldots ., 01\}$ & 492 & 491.88 & 49.53 \\
\hline & $\{98, \ldots, 02\}$ & 497 & 496.85 & 49.43 \\
\hline & $\{97, \ldots, 03\}$ & 498 & 497.85 & 49.37 \\
\hline & $\{95, \ldots, 05\}$ & 514 & 513.81 & 49.42 \\
\hline & $\{90, \ldots, \ldots, 10\}$ & 558 & 557.85 & 49.75 \\
\hline
\end{tabular}

Note: $\{97, \ldots \ldots, 03\}$ implies $\{97,98,99,00,01,02,03\}$, etc. 


\section{References}

Aggarwal, R. and B. M. Lucey, 2006. Psychological barriers in gold prices?, Review of Financial Economics, in press.

Benford, F., 1938. The law of anomalous numbers, Proceedings of the American Philosophical Society $78,551-572$.

Cyree, K. B., D. L. Domian, D. A. Louton and E. J. Yobaccio (1999). Evidence of psychological barriers in the conditional moments of major world stock indices, Review of Financial Economics 8, 73-91.

De Ceuster, M. K. J., G. Dhaene and T. Schatteman, 1998. On the hypothesis of psychological barriers in stock markets and Benford's law, Journal of Empirical Finance 5, 263-279.

Donaldson, R. G. and H. Y. Kim, 1993. Price barriers in the Dow Jones industrial average, Journal of Financial and Quanatative Analysis 28, 313-330.

Geyer, C. L. and P. P. Williamson, 2004. Detecting fraud in data sets using Benford's law, Communications in Statistics B 33, 229-246.

Giles, D. E. A., 2006. Benford's law and naturally occurring prices in certain eBay auctions, Applied Economics Letters, in press.

Koedijk, K. G. and P. A. Stork (1994). Should we care? Psychological barriers in stock markets, Economics Letters 44, 427-32.

Kuiper, N. H., 1959. Alternative proof of a theorem of Birnhaum and Pyke, Annals of Mathematical Statistics 30, 251-252.

Ley, E. and H. Varian, 1994. Are there psychological barriers in the Dow-Jones index?, Applied Financial Economics 4, 217-224.

Newcomb, S. (1881), Note on the frequency of use of different digits in natural numbers, American Journal of Mathematics 4, 39-40.

Nigrini, M. J., 1992. The detection of income tax evasion through an analysis of digital frequencies, Ph.D. Dissertation, University of Cincinnati.

Nigrini, M. J. and L. I. Mittermaier, 1997. The use of Benford's law as an aid in analytical procedures, Auditing: A Journal of Practice and Theory 16, 52-67.

Pinkham, R. S., 1961. On the distribution of first significant digits, Annals of Mathematical Statistics 32 1223-1230. 